\title{
Activation in Human MT/MST by Static Images with Implied Motion
}

\author{
Zoe Kourtzi and Nancy Kanwisher
}

Massachusetts Institute of Technology

\begin{abstract}
A still photograph of an object in motion may convey dynamic information about the position of the object immediately before and after the photograph was taken (implied motion). Medial temporal/medial superior temporal cortex (MT/MST) is one of the main brain regions engaged in the perceptual analysis of visual motion. In two experiments we examined whether MT/MST is also involved in representing
\end{abstract}

The perception of motion is critical for our ability to interact with a dynamic environment. Neurophysiological studies in monkeys (for example, Britten, Newsome, Shalden, Celebrini, \& Movshon, 1996; Dubner \& Zeki, 1971; Maunsell \& Van Essen, 1983; Van Essen, Maunsell, \& Bixby, 1981) and imaging studies in humans (Dupont, Orban, De Bruyn, Verbruggen, \& Mortelmans, 1994; Tootell et al., 1995b; Watson et al., 1993; Zeki et al., 1991) have shown that a network of brain regions in the primate visual system is devoted to the important task of analyzing visual motion. One of the main regions involved in motion processing is the extrastriate visual area medial temporal/medial superior temporal cortex (MT/MST). Recent imaging studies have shown that MT/MST is involved not only in the analysis of the continuous coherent motion of a physical stimulus, but also in the processing of apparent motion (Goebel, Khorram-Sefat, Muckli, Hacker, \& Singer, 1998; Kaneoke, Bundou, Koyama, Suzuki, \& Kakigi, 1997), illusory motion (Tootell et al., 1995a; Zeki, Watson, \& Frackowiak, 1993) and imagined motion (Goebel et al., 1998; O'Craven \& Kanwisher, 1997).

Most physiological and imaging studies of MT/MST have used stimuli such as moving dots and gratings. These stimuli consist of multiple sequential frames, each of which contains information about the position of the stimulus in space at a specific moment in time. However, in naturally occurring motion an instantaneous frame from a continuous-motion sequence often contains information not only about the current position of the objects in the frame, but also about their motion trajectory. Based on our knowledge of how animate and implied motion from static images. We found stronger functional magnetic resonance imaging (fMRI) activation within MT/MST during viewing of static photographs with implied motion compared to viewing of photographs without implied motion. These results suggest that brain regions involved in the visual analysis of motion are also engaged in processing implied dynamic information from static images.

inanimate objects move, we can infer the position of objects in a subsequent moment in time. Consider the "action photograph" in Figure 2a: The motion implied in this photograph allows us to anticipate the future position of the actor a moment after the photograph was taken. Psychophysical studies have demonstrated that observers extract this kind of dynamic information by extrapolating an object's future position from the motion implied in a static photograph. Specifically, when asked to judge whether two still photographs are the same or different, subjects often wrongly categorize them as identical when the second one is a photograph of the same event depicted in the first photograph, but taken a moment later in time (Freyd, 1983). These studies suggest that dynamic information can be extracted from still photographs even when the task does not require it.

The current studies were designed to test whether brain areas known to be involved in the analysis of physical stimulus motion are also engaged in processing dynamic information from static images with implied motion. To this end, we used functional magnetic resonance imaging (fMRI) to localize area MT/MST in each subject individually, and then measured activity in this area, while the subjects observed static photographs of human athletes in action (implied motion images) or of athletes at rest (no implied motion). In two further conditions in the same scans, subjects viewed another set of photographs of houses (an example of a stimulus conveying no dynamic information) and photographs of people at rest (to control for the possibility that the athletes at rest could be associated with information about action 
since athletes were also presented the implied motion condition). Half the subjects viewed these four different kinds of photographs passively. To ensure attention to stimuli from all conditions, the other half of the subjects performed a "1-back" repetition detection task on the same sequences. In a second experiment, we tested the response of area MT/MST to photo- graphs of animals and nature scenes that either depicted implied motion or did not.

\section{RESULTS}

The localizer scans (low contrast moving vs. stationary rings) successfully localized each subject's MT/MST in

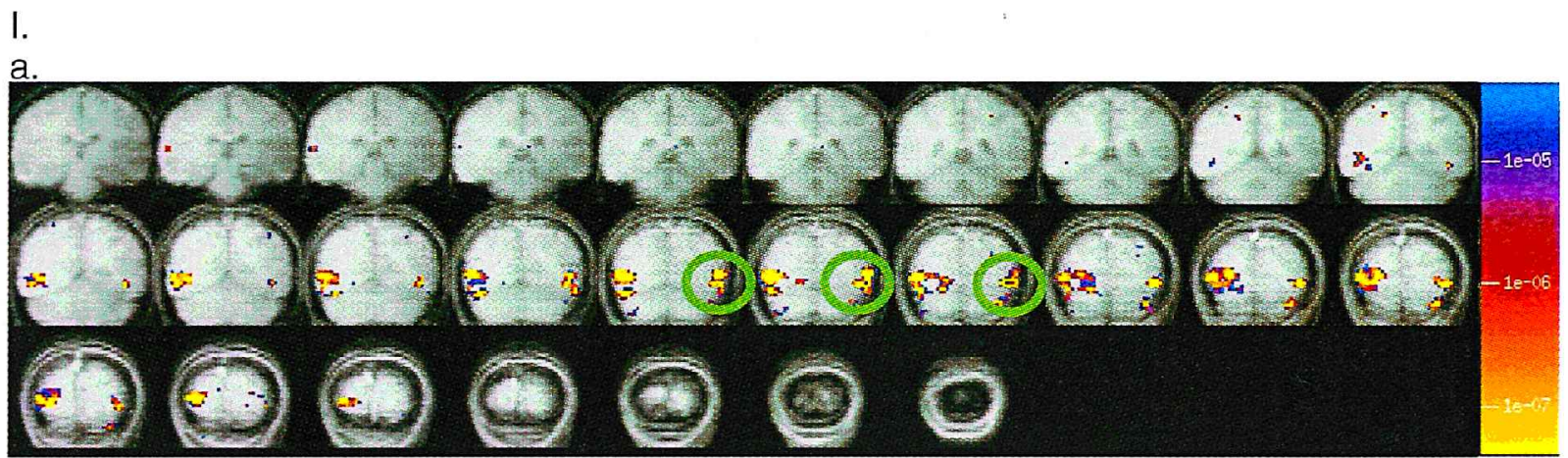

b.

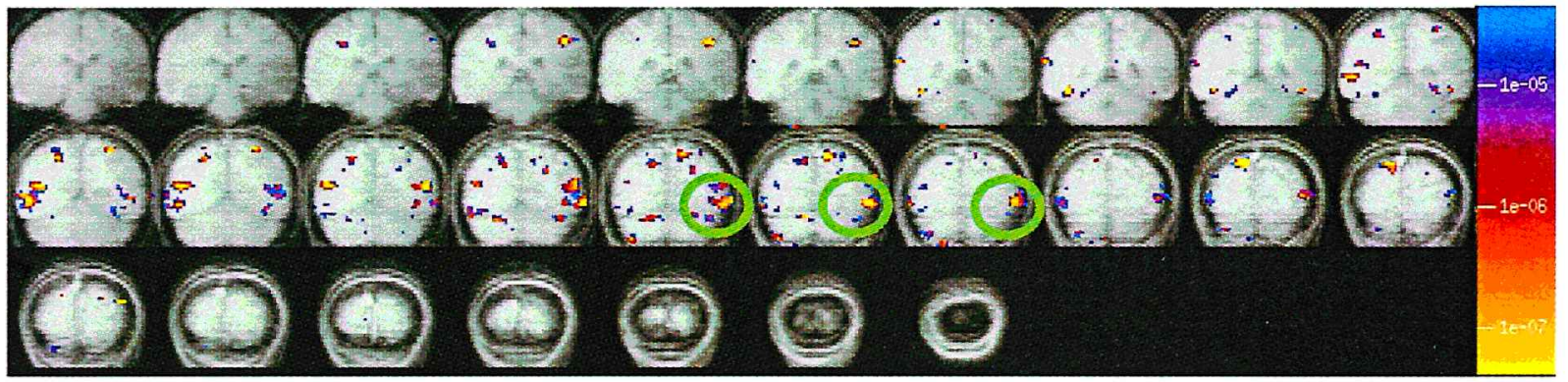

II.

a.

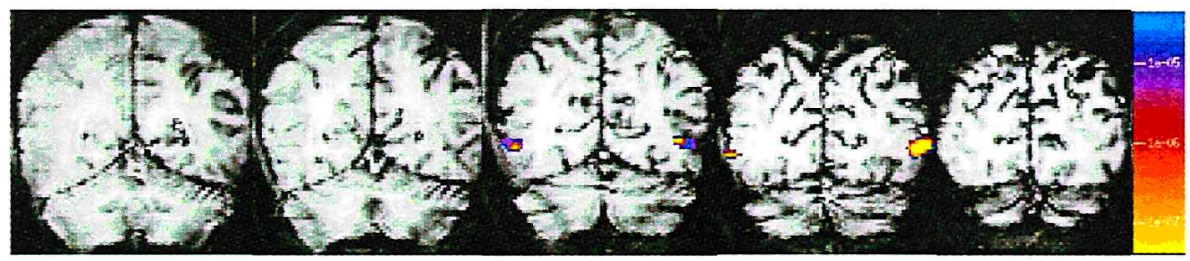

b.

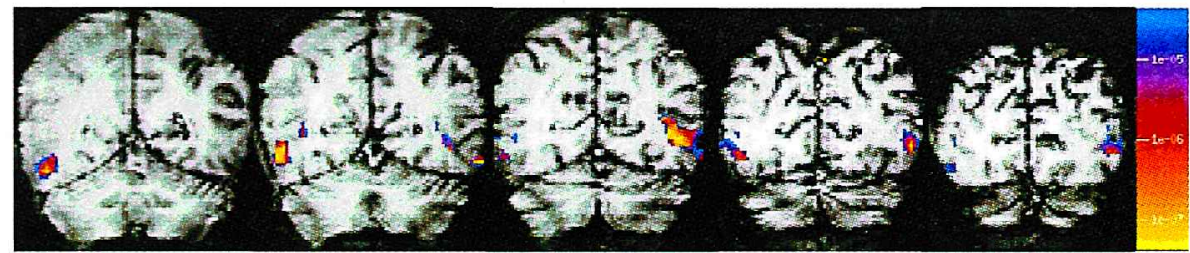

Figure 1. Functional data are overlaid on a high-resolution T1-weighted anatomical image for each slice. Right hemisphere appears on the left. Significance levels reflect the results of $t$-tests on the MR signal intensity $\left(p<.10^{-7}\right.$, equivalent to $p<.10^{-1}$ after Bonferroni correction). I. Group analysis on functional data from 5 subjects (coregistered in Talairach space) showing regions responding significantly to (a) moving vs. stationary rings and (b) images with implied motion vs. images without implied motion (Experiment 1). The green circles indicate regions activated significantly for both moving vs. stationary rings and images with implied motion vs. images without implied motion. II. Five slices from one subject showing activation for viewing of (a) moving vs. stationary rings and (b) images with implied motion vs. images without implied motion (Experiment 1). 
the lateral occipital region (Figure 1) consistent with prior reports (for example, Tootell et al., 1995b). For each subject, this region served as the region of interest (ROI) from which the response was extracted for each of the experimental conditions for the same subject. The response for each condition and subject was quantified as the percent signal change (PSC) from the fixation baseline condition. The average PSC across subjects for each condition and the time course of signal intensity averaged across subjects are shown in Figure 2 for Experiment 1 and Figure 3 for Experiment 2.

For the first experiment, a two-way ANOVA (Stimulus Type $\times$ Task) on the PSC for each condition across subjects with Stimulus Type (implied motion athletes, no implied motion athletes, people at rest, houses) as the within-subjects variable and Task (passive, 1-back) as the between-subjects variable showed a significant main effect of Stimulus Type $(F(3,18)=20.1, p<.001)$. There was no main effect of Task $(F(1,18)<1)$, and no interaction of Stimulus Type and Task $(F(3,18)=1.2, p>.3)$. The PSC in MT/MST was significantly greater for images of athletes with implied motion vs. athletes without implied motion in both the passive $(t(3)=3.5, p<.05)$ and the 1-back $(t(3)=4.5, p<.05)$ tasks. The PSC in MT/ MST during viewing of athletes without implied motion was not significantly different from that for people at rest $(t(7)=0.6, p>.5)$.

The similar patterns of activation in MT/MST across passive viewing and 1-back tasks suggest that the observed activation is not likely to be due to differences in task difficulty or attentional allocation across conditions. Indeed, the behavioral data from the 1-back task suggest that this matching task was at least as difficult for images without implied motion as for images with implied motion. Specifically, across three out of the four subjects (the behavioral data for one subject were lost due to a computer error), the average percent correct detection

a

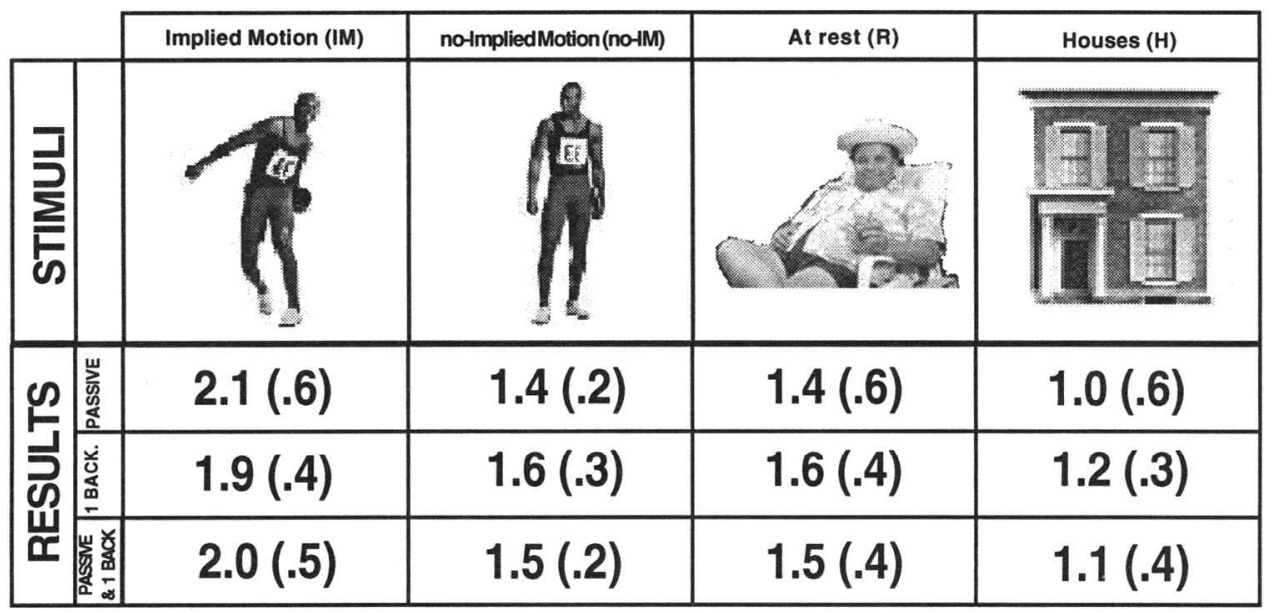

b

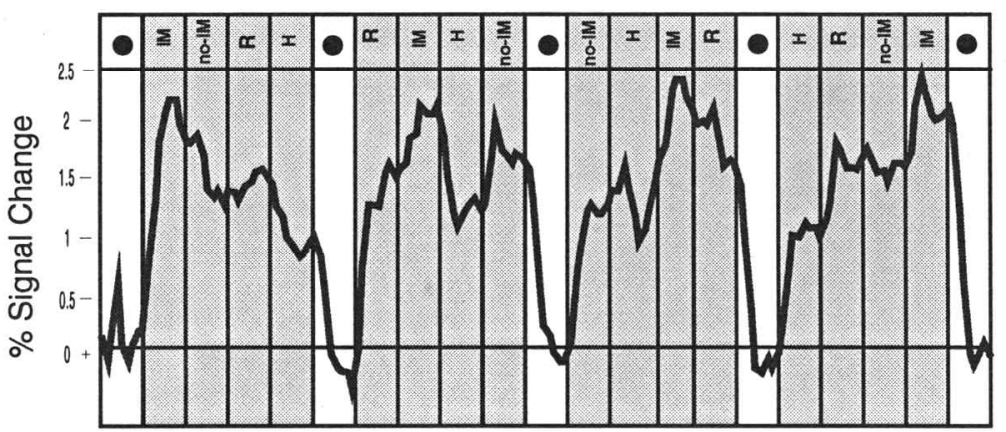

Figure 2. Results of Experiment 1: (a) An example stimulus from each condition. Average percent signal increase (from the fixation baseline) and standard deviations across subjects for each stimulus type in MT observed for each task (passive viewing, 1-back), as well as the average across tasks. (b) The time course of the percent change in MR signal intensity (from the fixation baseline) in MT over the period of the scan. Black dot indicates fixation, IM: images of athletes with implied motion, no-IM: images of athletes without implied motion, R: people at rest, H: houses. 


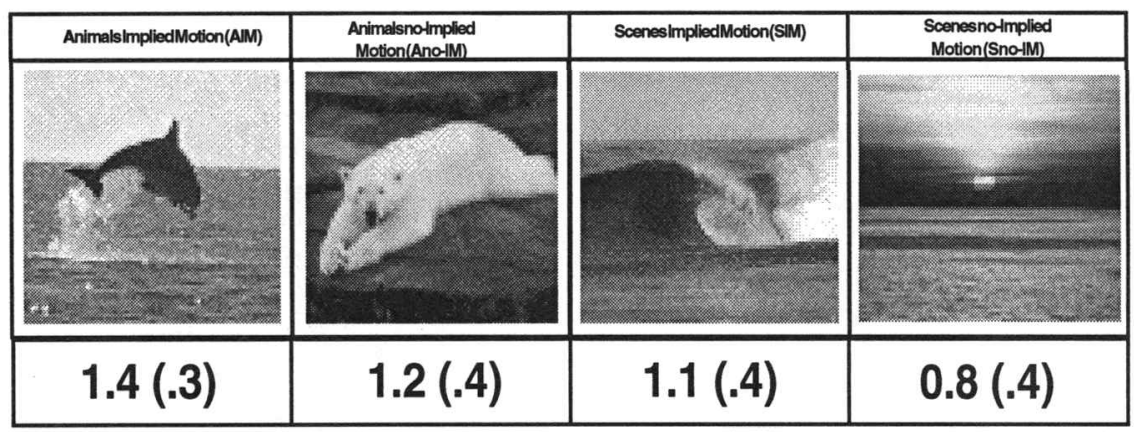

b

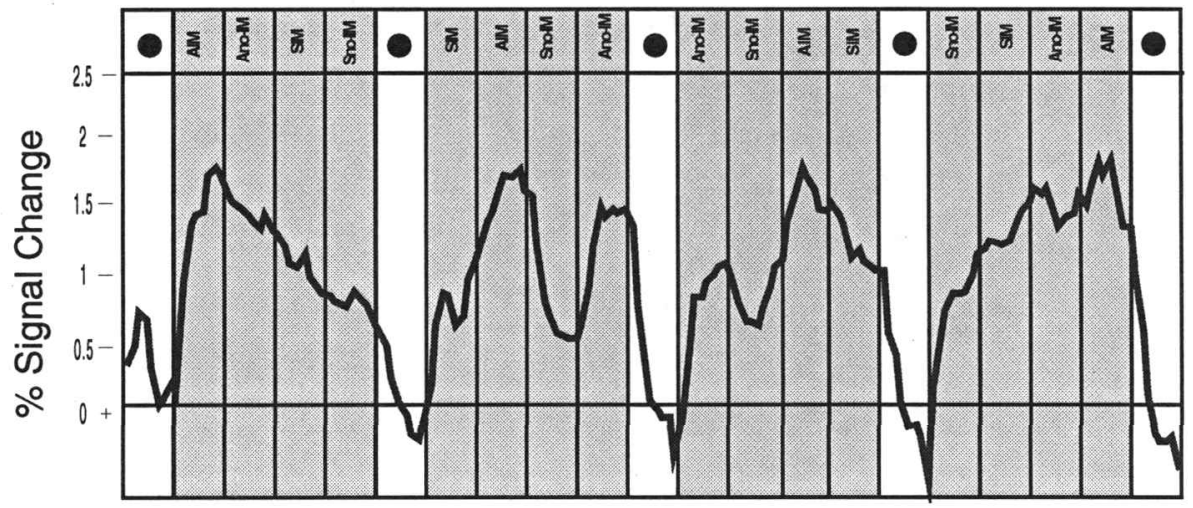

Figure 3. Results of Experiment 2: (a) An example stimulus from each condition. Average percent signal change and standard deviations across subjects for each stimulus type in MT. (b) The time course of the percent change in MR signal intensity in MT over the period of the scan. Black dot indicates fixation, AIM: images of animals with implied motion, Ano IM: images of animals without implied motion, SIM: images of nature scenes with implied motion, Sno IM: images of nature scenes without implied motion.

and total number of false alarms (in parentheses) over four epochs for each condition were: Implied motion $90 \%$ (1), no implied motion $82 \%$ (1), people at rest 93\% (2) and Houses 74\% (1).

The data from Experiment 2 were analyzed by a twoway repeated ANOVA (Condition $\times$ Stimulus Type) with Condition (implied motion vs. no implied motion) and Stimulus Type (animals vs. nature scenes) as repeated measures variables. A main effect of Condition $(F(1$, $3)=268.5, p<.001)$ was observed. No main effect of Stimulus Type $(F(1,3)=6.847, p=.079)$ nor a significant interaction of Stimulus Type and Condition $(F(1,3)<1)$ was observed. The PSC within MT/MST was significantly greater for implied than for no implied motion conditions for both animals $(t(3)=3.7, p<.05)$ and nature scenes $(t(3)=6.1, p<.01)$.

In order to look at regions of the brain beyond MT/ MST, Kolmogorov-Smirnov statistics were run on each voxel scanned in each subject in Experiment 1, testing whether that voxel showed stronger activation for (i) moving vs. stationary rings in the localizer runs, and (ii) implied motion athletes vs. no implied motion athletes.
For all subjects, the lateral occipital regions that showed significant activation for moving vs. stationary rings in the localizer task overlapped with regions showing significant activation for implied motion vs. no implied motion in the experimental runs. However, for all subjects the implied motion vs. no implied motion athletes comparison also activated other regions contiguous to MT/MST extending medially, anteriorly, and posteriorly. Six out of the eight subjects in Experiment 1 also showed significant activation for implied motion vs. no implied motion in the region of the superior temporal sulcus. Activations in these regions were also observed in $t$-test group analyses of five subjects coregistered into Talairach space (Talairach \& Tournoux, 1988). (Three subjects could not be coregistered due to poor resolution in the frontal regions of the brain as a result of surface coil usage.) These analyses (see Figure 1) showed significantly stronger activations $\left(p<.10^{-7}\right.$, equivalent to $p<.10^{-1}$ after Bonferroni correction) for moving compared to static rings in MT/MST and for implied motion vs. no implied motion. 


\section{DISCUSSION}

Our results suggest that cortical areas involved in the analysis of physical stimulus motion can be also engaged automatically by static images that merely imply motion. Specifically, passively observed static snapshots of objects in action activate human motion areas (MT/ MST) more than static images of objects without implied motion. These results are observed for images implying animate motion, such as humans or animals in action, as well as inanimate motion, such as active nature scenes.

It is unlikely that these results can be explained by low-level differences among the images in the different conditions (for example, differences in the location of the luminance edges). The activation in MT/MST was systematically greater for implied than no implied motion across eight very different stimulus categories used in the two experiments. Furthermore, it is unlikely that the modulation of activity in MT/MST is related to differences in image flicker (each photograph was displayed for $300 \mathrm{msec}$ followed by a 500-msec blank interval, followed by the next stimulus), since this flicker occurred in all of our stimulus conditions.

These results raise numerous questions about the analysis of object motion in the human brain. That is, is MT/MST involved in extracting implied motion information, or is it influenced by such processes occurring elsewhere in the brain? It seems unlikely that the perceptual analyses involved in the inference of motion from still images could be computed within MT/MST. Neurophysiological and imaging studies have strongly supported the role of MT/MST in the analysis of stimulus motion but not in processes such as object recognition. Inferring motion from still images depends on object categorization and knowledge about the repertoire of behavior different objects can exhibit. It seems most likely that such high-level perceptual inferences occur elsewhere in the brain and modulate activity in MT/MST in a top-down fashion. Thus, the observed activations may reflect an expectancy of object motion that could be represented or influence representations in areas involved in processing physical stimulus motion (that is, MT/MST).

Consistent with this hypothesis, the activation for implied vs. no implied motion extended beyond MT/ MST to several contiguous regions, as shown in Figure 1. These results are consistent with recent studies suggesting that other areas extending posterior and superior or anterior and inferior to MT/MST are also involved in motion analysis (De Jong, Shipp, Skidmore, Frackowiak, \& Zeki, 1994; Dupont et al., 1994; Shipp, De Jong, Zihl, Frackowiak, \& Zeki, 1994; Watson et al., 1993; ). Previous research has shown activation anterior and medial to MT for passive viewing of images of illusory motion (Zeki, Watson, \& Frackowiak, 1993), tool naming (Martin, Wiggs, Ungerleider, \& Haxby, 1996) and the genera- tion of action words (Martin, Haxby, Lalonde, Wiggs, \& Ungerleider, 1995). Recent imaging studies have shown activation for motion boundaries in areas V3A (Tootell et al., 1997) and KO (Orban, Dupont, De Bruyn, Vogels, Vandenberghe, \& Mortelmans, 1995; Van Oostende, Sunaert, Van Hecke, Marchal, \& Orban, 1997) extending posterior and medial to MT along the occipital surface. The activations observed in our subjects in the vicinity of the superior temporal sulcus are also consistent with previous studies showing activation in the superior temporal sulcus for motion imagery (Goebel et al., 1998), and viewing of biological motion stimuli (Bonda, Petrides, Ostry, \& Evans, 1996; Puce, Allison, Bentin, Gore, \& McCarthy, 1998).

Finally, several prior findings support the hypothesis that the current results reflect top-down influences of high-level perceptual inferences on MT/MST. Both single unit (Treue \& Maunsell, 1996) and fMRI studies (Beauchamp, Cox, \& DeYoe, 1997; Corbetta, Miezin, Dobmeyer, Shulman, \& Petersen, 1990, 1991; O'Craven, Rosen, Kwong, Treisman, \& Savoy, 1997) have demonstrated that the response of MT/MST to moving stimuli can be strongly modulated by visual attention. Also, activity in MT/MST has been demonstrated even when subjects close their eyes and merely imagine moving compared to stationary arrays (Goebel et al., 1998; O'Craven \& Kanwisher, 1997).

While the present work is consistent with these previous studies, suggesting that activation in MT/MST can be modulated in a top-down fashion, we show here for the first time that such top-down effects can occur automatically. That is, dynamic information implicit in the image was extracted and influenced activity in MT/ MST, even though subjects were not asked or required to perceive, attend to, or imagine motion.

One possible interpretation of our findings is that inferring motion may involve or result in motion imagery. Another interpretation is that the processing of a particular object category (for example, animals) may lead to activation of regions involved in processing properties highly associated with that object category (for example, motion) (Chao, Haxby, Lalonde, Ungerleider, \& Martin, 1998; Martin et al., 1996). Consistent with the second hypothesis, the current findings show that activation in MT/MST is significantly higher for images of people, even people at rest than for images of houses.

More broadly, the current results support an emerging view of extrastriate cortex as playing a crucial role not only in visual perception, but also in visual cognition.

\section{METHODS}

\section{Subjects}

Ten right-handed MIT students participated in Experiment 1 , four in the passive viewing condition and six in the 1-back matching condition. Two subjects tested on 
the 1-back matching condition were excluded from the analysis due to excessive head motion. Another six righthanded MIT students participated in Experiment 2. Two subjects were excluded from the analysis in this condition due to excessive head motion.

\section{Materials and Design}

The stimuli used for functionally localizing MT were low contrast moving vs. stationary concentric rings as described in Tootell et al. (1995a). For the experimental conditions, stimuli were $300 \times 300$ pixel digitized grayscale photographs. Experiment 1 involved a mixed design, with Stimulus Type a within-subject variable (with four levels: photographs of athletes with implied motion, athletes without implied motion, people at rest, and houses) and Task a between-subjects factor (with two levels: passive viewing vs. 1-back repetition detection). Experiment 2 involved two orthogonal factors crossed within subjects: Stimulus Type (animals vs. scenes) and Condition (implied motion vs. no implied motion).

\section{Procedure}

Each subject was run on two or more functional MT localizer scans with low contrast moving vs. stationary concentric rings (as described in Tootell et al., 1995a). Then each subject was run on four scans of the experimental test materials. For the passive viewing conditions, the subjects were asked to observe the images carefully while fixating a dot in the center of the image. (Monitoring of eye movements outside the scanner for three subjects that participated in Experiment 1 and three subjects that participated in Experiment 2 showed that the number of eye movements was very small in all conditions and did not differ significantly across conditions.) For the 1-back matching condition, subjects were instructed to press a button whenever they saw two identical pictures in a row. Two or more repetitions occurred in each epoch.

Each scan lasted $5 \mathrm{~min}$ and $36 \mathrm{sec}$ and consisted of sixteen 16-sec epochs with fixation periods interleaved, as shown in Figures 2 and 3. Twenty different photographs of the same type were presented in each epoch. Each photograph was presented for $300 \mathrm{msec}$ with a blank interval of 500 msec between photographs. Each of the four stimulus types in each experiment were presented in four different epochs within each scan, in a design that balanced for the order of conditions, as shown in Figures 2 and 3.

\section{MRI Acquisition}

Scanning was done on the $3 \mathrm{~T}$ scanner (modified by ANMR for Echo Planar Imaging) at the MGH-NMR Center in Charlestown, MA. A custom bilateral surface coil (built by J. Thomas Vaughan) provided a high signalto-noise ratio in posterior brain regions. A bite-bar was used to minimize head motion. Standard imaging procedures (Gradient Echo pulse sequence, TR, 2 sec; TE, $30 \mathrm{msec}$; flip angle, $90^{\circ}$; $180^{\circ}$ offset, $25 \mathrm{msec}$ ) were used as described previously (Tong, Nakayama, Vaughan, \& Kanwisher, 1998). Twelve 6-mm-thick near-coronal slices were oriented parallel to the brainstem and covered the occipital lobe as well as the posterior portions of the temporal and the parietal lobes. One hundred sixtyeight functional images were collected for each slice in each scan.

\section{Data Analysis}

Each subject's MT/MST was identified from the average of the functional localizer scans as the set of all contiguous voxels in the vicinity of the ascending limb of the inferior temporal sulcus (Tootell et al., 1995b; Watson et al., 1993; Zeki et al., 1991) that showed significantly stronger activation to moving compared to static low-contrast concentric rings on a Kolmogorov-Smirnov test at the level of $p<.0001$ (uncorrected). In principle, significant differences in KolmogorovSmirnov statistics can reflect differences in the variance only, rather than in the means across conditions (Aguirre, Zarahn, \& D'Esposito, 1998). However, the fact that the region selected by this procedure did, indeed, respond more strongly during the moving than stationary conditions was confirmed by subsequent analyses. In particular, $t$-tests across subjects revealed that the percent signal change in the selected ROIs was higher for moving than stationary conditions (a difference of $0.9 \%,(t(7)=7.3, p<.001)$ for Experiment 1 , and $0.7 \%(t(3)=6.3, p<.01)$ for Experiment 2$)$. Moreover, as shown in Figure $1, t$-tests on the averaged group data for five subjects showed significantly stronger activation to moving compared to static rings $\left(p<.10^{-7}\right.$, equivalent to $p<.10^{-1}$ after Bonferroni correction).

For the analysis of the experimental scans, the time course of MR signal intensity was extracted from each subject's MT/MST by averaging the data from all voxels within the ROI. The average percent signal change in MT/MST was calculated for each subject and stimulus type, using the average signal intensity during fixation epochs for the same subject, experiment, and task as a baseline. Because the fMRI response typically lags four to six seconds after the neural response, our data-analysis procedure treated the first image in each epoch as belonging to the condition of the preceding epoch, and omitted the next two images (during the transition between epochs) from the analysis.

An ANOVA across subjects was run on the average percent signal change in each of the conditions in each experiment. Because data were analysed within inde- 
pendently defined ROIs for MT/MST, no correction for multiple voxelwise comparisons was required.

\section{Acknowledgments}

We would like to thank Ted Adelson and Maggie Shiffrar for their helpful comments and suggestions on this project, and Bruce Rosen, and many people at the MGH-NMR Center for technical assistance and support. We would also like to thank Paul Downing and Russell Epstein for their comments on previous versions of this manuscript. This research was supported by NIMH Grant 56037, and a Human Frontiers grant to Nancy Kanwisher. Some of the results discussed in this manuscript were first presented at the 1998 meeting of the Society for Neuroscience in Los Angeles, CA and the 1998 meeting of the Psychonomic Society in Dallas, TX.

Reprint requests should be sent to Zoe Kourtzi, Dept. of Brain and Cognitive Science, MIT, NE20-4043, 77 Massachusetts Ave, Cambridge, MA, 02139-4307, or via e-mail: zoe@psyche.mit.edu.

\section{REFERENCES}

Aguirre, G. K., Zarahn, E., \& D'Esposito, M. (1998). A critique of the use of the Kolmogorov-Smirnov (KS) statistic for the analysis of BOLD fMRI data. Magnetic Resonance in Medicine, 39, 500-505.

Beauchamp, M. S., Cox, R. W., \& DeYoe, E. A. (1997). Graded effects of spatial and featural attention on human-area MT and associated motion-processing areas. Journal of Neurophysiology, 78, 516-520.

Bonda, E., Petrides, M., Ostry, D., \& Evans, A. (1996). Specific involvement of human-parietal systems, and the amygdala, in the perception of biological motion. The Journal of Neuroscience, 16, 3737-3744.

Britten, K. H., Newsome, W. T., Shalden, M. N., Celebrini, S., \& Movshon, J. A. (1996). A relationship between behavioral choice and the visual responses of neurons in macaque MT. Visual Neuroscience, 13, 87-100.

Chao, L. L., Haxby, J. V., Lalonde, F. M., Ungerleider, L. G., \& Martin, A. (1998). Pictures of animals and tools deferentially engage object form-related and motion-related brain regions. Paper presented at the 28th Annual Meeting of the Society for Neuroscience, LA, CA.

Corbetta, M., Miezin, F. M., Dobmeyer, S., Shulman, G. L., \& Petersen, S. E. (1990). Attentional modulation of neural processing of shape, color, and velocity in humans. Science, 248, 1556-1559.

Corbetta, M., Miezin, F. M., Dobmeyer, S., Shulman, G. L., \& Petersen, S. E. (1991). Selective and divided attention during visual discriminations of shape, color, and speed: Functional anatomy by positron emission tomography. Journal of Neuroscience, 11, 2383-2402.

De Jong, B. M., Shipp, S., Skidmore, B., Frackowiak, R. S. J., \& Zeki, S. (1994). The cerebral activity related to visual perception of forward motion in depth. Brain, 117, 10391054 .

Dubner, R., \& Zeki, S. M. (1971). Response properties and receptive fields of cells in an anatomically-defined region of the superior temporal sulcus in the monkey. Brain Research, 35, 528-532.

Dupont, P., Orban, G. A., De Bruyn, B., Verbruggen, A., \& Mortelmans, L. (1994). Many areas in the human brain respond to visual motion. Journal of Neurophysiology, 72, 1420-1424.

Freyd, J. (1983). The mental representation of movement when static stimuli are viewed. Perception and Psychophysics, 33, 575-581.

Goebel, R., Khorram-Sefat, D., Muckli, L., Hacker, H., \& Singer, W. (1998). The constructive nature of vision: Direct evidence from functional magnetic resonance imaging studies of apparent motion and motion imagery. European Journal of Neuroscience, 10, 1563-1573.

Kaneoke, Y., Bundou, M., Koyama, S., Suzuki, H., \& Kakigi, R. (1997). Human cortical area responding to stimuli in apparent motion. NeuroReport, 8, 677-682.

Martin, A., Haxby, J. V., Lalonde, F. M., Wiggs, C. L., \& Ungerleider, L. G. (1995). Discrete cortical regions associated with knowledge of color and knowledge of action. Science, 270, $102-105$.

Martin, A., Wiggs, C. L., Ungerleider, L. G., \& Haxby, J. V. (1996). Neural correlates of category-specific knowledge. Nature, 379, 649-652.

Maunsell, J. H., \& Van Essen, D. C. (1983). The connections of the middle temporal-visual area (MT), and their relationship to a cortical hierarchy in the macaque monkey. Journal of Neuroscience, 3, 2563-2586.

O'Craven, K. M., \& Kanwisher, N. G. (1997). Visual imagery of moving stimuli activates area MT/MST. Paper presented at the 27 th Annual Meeting of the Society for Neuroscience, New Orleans, LA.

O'Craven, K. M., Rosen, B. R., Kwong, K. K., Treisman, A., \& Savoy, R. L. (1997). Voluntary attention modulates fMRI activity in human MT-MST. Neuron, 18, 591-598.

Orban, G. A., Dupont, P., De Bruyn, B., Vogels, R., Vandenberghe, R., \& Mortelmans, L. (1995). A motion area in human visual cortex. Proceedings of the National Academy of Science, 92, 993-997.

Puce, A., Allison, T., Bentin, S., Gore, J. C., \& McCarthy, G. (1998). Temporal-cortex activation in human subjects viewing eye and mouth movements. Journal of Neuroscience, $18,2188-2199$.

Shipp, S., de Jong, B. M., Zihl, J., Frackowiak, R. S. J., \& Zeki, S. (1994). The brain activity related to residual motion vision in a patient with bilateral lesions of V5. Brain, 117, 1023-1038.

Talairach, J., \& Tournoux, P. (1988). Co-planar stereotaxic atlas of the buman brain. New York: Thieme Medical.

Tong, F., Nakayama, K., Vaughan, J. T., \& Kanwisher, N. (1998). Binocular rivalry and visual awareness in human extrastriate cortex. Neuron, 21, 753-759.

Tootell, R. B. H., Mendola, J. D., Hadjikhani, N. K., Ledden, P. J., Liu, A. K., Reppas, J. B., Sereno, M., I., \& Dale, A. M. (1997). Functional analysis of V3A and related areas in human visual cortex. The Journal of Neuroscience, 15, 70607078.

Tootell, R. B. H., Reppas, J. B., Dale, A. M., Look, R. B., Sereno, M., I., Malach, R., Brady, T. J., \& Rosen, B. R. (1995a). Visual motion after effect in human cortical area MT revealed by functional magnetic resonance imaging. Nature, 11, 139141.

Tootell, R. B. H., Reppas, J. B., Kwong, K. K., Malach, R., Born, R. T., Brady, T. J., Rosen, B. R., \& Belliveau, J. W. (1995b). Functional analysis of human MT and related-visual cortical areas using magnetic resonance imaging. The Journal of Neuroscience, 15, 3215-3230.

Treue, S., \& Maunsell, J. H. R. (1996). Attentional modulation of visual motion processing in cortical areas MT and MST. Nature, 382, 539-541.

Van Essen, D. C., Maunsell, J. H., \& Bixby, J. L. (1981). The middle temporal visual area in the macaque: myeloarchitecture, connections, functional properties and topographic organization. Journal of Comparative Neurology, 199, 293326. 
Van Oostende, S., Sunaert, S., Van Hecke, P., Marchal, G., \& Orban, G. A. (1997). The Kinetic Occipital (KO) region in man: An fMRI study. Cerebral Cortex, 7, 690-701.

Watson, J. D. G., Myers, R., Frackowiak, R. S. J., Hajnal, J. V., Woods, R. P., Mazziotta, J. C., Shipp, S., \& Zeki, S. (1993). Area V5 of the human brain: Evidence from a combined study using positron emission tomography and magnetic resonance imaging. Cerebral Cortex, 3, 79-94.
Zeki, S., Watson, J. D. G., Lueck, C., J., Friston, K. J., Kennard, C., \& Frackowiak, R. S. J. (1991). A direct discrimination of functional specialization in human visual cortex. Journal of Neuroscience, 11, 641-649.

Zeki, S., Watson, J. D. G., \& Frackowiak, R. S. J. (1993). Going beyond the information given: The relation of illusory visual motion to brain activity. Proceedings of the Royal Society of London B, 252, 215-222. 